\title{
The de Broglie Waves and Joule-Lenz Law Applied in Examining the Electron Transitions in Small Quantum Systems
}

\author{
Stanislaw Olszewski \\ Institute of Physical Chemistry, Polish Academy of Sciences, Warsaw, Poland \\ Email: olsz@ichf.edu.pl
}

How to cite this paper: Olszewski, S. (2019) The de Broglie Waves and Joule-Lenz Law Applied in Examining the Electron Transitions in Small Quantum Systems. Journal of Modern Physics, 10, 176-194. https://doi.org/10.4236/jmp.2019.102014

Received: January 12, 2019

Accepted: February 24, 2019

Published: February 27, 2019

Copyright $\odot 2019$ by author(s) and Scientific Research Publishing Inc. This work is licensed under the Creative Commons Attribution International License (CC BY 4.0).

http://creativecommons.org/licenses/by/4.0/

\section{cc) (i) Open Access}

\begin{abstract}
A transformation of the electron states-say those enclosed in a potential box-into the de Broglie waves done in the paper, enabled us to calculate the energy change between two quantum levels as a function of the specific heat and difference of the temperature between the states. In consequence, the energy difference and that of entropy between the levels could be examined in terms of the appropriate classical parameters. In the next step, the time interval necessary for the electron transition between the levels could be associated with the classical electrodynamical parameters like the electric resistance and capacitance connected with the temporary formation of the electric cell in course of the transition. The parameters characterizing the mechanical inertia of the electron were next used as a check of the electrodynamical formulae referring to transition.
\end{abstract}

\section{Keywords}

The de Broglie Waves, Specific Heat, and Energy as Well as Entropy Transfer in Small Quantum Systems, Time Interval of the Electron Transition Associated with Parameters of the Classical Electrodynamics

\section{Introduction}

The spectacular results obtained by Planck (see e.g. [1]) at the very beginning of the quantum theory allowed him to couple the energy changes of the quantum oscillators with the temperature and entropy. The Planck's results concerning the probabilistic aspects of the changes of quantum oscillators were next generalized by Einstein [2]. Further development of the quantum theory led mainly to an accurate calculation of the stationary quantum states leaving the problem of 
the electron transitions, and their properties, approximately on the level presented by Einstein. A step forwards was here the wave functions of the stationary states applied in calculating the transition probabilities between different quantum levels.

Another feature of the probabilistic Einstein theory was the assumption that a large, though rather undefined, number of the quantum objects should enter a given transition. This difficulty seems to be not involved in the Planck's approach where the number of the states which participate in transition can be definited and not necessarily large. This property allows us to consider also transitions in which the number of participating objects is relatively small. Moreover, when the Joule-Lenz classical approach [3] is applied on the quantum footing [4], the intensity of the energy emission can be estimated for a transition of a single particle without any reference to the probabilistic theory.

In effect, the aim of the present paper became to examine a single electron transition in small quantum systems on both probabilistic and non-probabilistic footing. An analysis of the classical physical parameters of mechanics, thermodynamics and electrodynamics which can be connected with the transition seems to be then of use.

\section{Notion of Temperature Applied for a Small Number of Quantum Systems}

Historically the quantum theory began as a statistics of photons emitted in course of the black-body radiation. Here the notion of temperature does accompany systematically the presentation of the energy distribution among the quantum levels. Somewhat later a concurrent quantum theory by Schrödinger banned essentially the notion of temperature and that of particle oscillations from the basic idea of the quantum states: the spectrum of levels has been replaced by a set of discrete entities being in general essentially different in their individual properties. The temperature is then assumed to be close to zero.

Nevertheless, for less or more numerous ensembles of particles, a reference between the temperature and energy remained of importance. The point became especially sound for the case of very low temperatures. Here we have, on one side, the well-known Nernst law representing a reference between the vanishing temperature and similar behaviour of the specific heat, but on the other side, the Planck's doubt does exist concerning the validity of the thermodynamical laws in general in case when the system absolute temperature $T$ approaches zero [1].

The first step of the present paper is focused on a special problem of the electron specific heat. In general this heat is considered for large electron ensembles occupying a large number of the electron states. In consequence the problem is mainly reduced to the change of the Fermi level as an effect of the change of the temperature [5] [6] [7]. Such an approach seems to neglect details concerning the influence of the temperature on the individual levels. But the levels behaviour can be important also for small quantum systems, especially those having 
low dimensionality. In such systems the number of considered quantum levels can be limited to only few states. Their examination as a function of the change of $T$ becomes especially convenient when the dimensionality of the system is reduced together with the system size. An example can be a linear one-dimensional potential box represented by a very thin straight-linear tube. For, in this case, the system can be replaced by a set of the de Broglie waves. An advantage of such an approach is a transformation of the system into a frequency-and-temperature dependent ensemble similar to the photon system considered earlier by Planck.

\section{The One-Dimensional Free-Electron System and Its Wave-Like Properties}

The Schrödinger-like approach to such system is well known [8]. If we have the free-electron particles enclosed in a one-dimensional potential box of length $L$, their wave-mechanical properties are represented by the eigenenergies (see e.g. [8])

$$
E_{n}=\frac{n^{2} h^{2}}{8 m L^{2}}
$$

with the integer numbers

$$
n=1,2,3,4, \cdots
$$

indicating the quantum energy levels in (1), and the electron wave functions are

$$
\psi_{n}=\left(\frac{2}{L}\right)^{1 / 2} \sin \left(\frac{n \pi}{L} x\right)
$$

$x$ is a position coordinate extended along the box. Here any $\psi_{n}$ satisfies at the box boundaries the vanishing properties

$$
\psi_{n}(x=0)=\psi_{n}(x=L)=0 .
$$

The wave-like character can be attributed to the free-electron particles by taking into account, first, their kinetic energy in any state $n$, viz.

$$
\frac{m v_{n}^{2}}{2}=E_{n}
$$

where $v_{n}$ is the velocity of the particle in the box. This gives

$$
v_{n}=\left(\frac{2 E_{n}}{m}\right)^{1 / 2}=\frac{n h}{2 m L} .
$$

The particle is moving in one or another of the box directions with the speed of (6).

The de Broglie wave representing the particle in state $n$ has the frequency

$$
v_{n}=\frac{1}{\tau_{n}}=\frac{v_{n}}{2 L}=\frac{n h}{4 m L^{2}}
$$




$$
v_{n}=\frac{2 L}{\tau_{n}} .
$$

In (7) the time period $\tau_{n}$ of the wave oscillation is characterized by assuming that the particle is passing twice the way of the length $L$.

The next requirement is that the energy possessed by the electron in state $n$ is conserved by remaining equal to (1) also in the case of the de Broglie wave:

$$
E_{n}=\frac{n^{2} h^{2}}{8 m L^{2}}=\alpha n h v_{n}=\alpha n h \frac{n h}{4 m L^{2}}=\alpha \frac{n^{2} h^{2}}{4 m L^{2}} .
$$

We find from (9) put equal to (1) that

$$
\alpha=\frac{1}{2} .
$$

Therefore the quantum

$$
n h v_{n}=2 E_{n}
$$

represents a full (non-interacting) energy of the electron pair occupying-according to the Pauli principle-a single level $n$.

\section{Planck's Oscillator System as a Substitution of the Electron System}

In effect of Sec. 3 we obtain the energy of the system of electron particles replaced by the energy of a system of the oscillators: each pair of particles situated on the level $n$ is replaced by the oscillator having the energy $n h v_{n}$. The energy of the total system is therefore

$$
2 \sum_{n} E_{n}=\sum_{n} n h v_{n},
$$

where summation runs over the occupied states $n$.

A similar system of oscillators and its dependence on the temperature $T$ has been considered by Planck [1]. The probability that a single oscillator has at any time the energy

$$
\varepsilon_{n}=\varepsilon_{0}+n h v_{n}
$$

in the temperature bath of $T$ is:

$$
w_{n}=\mathrm{e}^{\frac{-n h v_{n}}{k T}}\left(1-\mathrm{e}^{-h v_{n} / k T}\right) \cong \mathrm{e}^{\frac{-n h v_{n}}{k T}} .
$$

The last step in (14) is due to the assumption of a very low $T$.

In effect - by neglecting $\varepsilon_{0}$ which is the energy constant characteristic for all oscillator levels $n$ - we have the energy

$$
E_{n}(T)=w_{n} n h v_{n}=\mathrm{e}^{\frac{-n h v_{n}}{k T}} n h v_{n} .
$$

The dependence of $E_{n}(T)$ on $T$ can be examined by taking different $n$. In general we can look for the contribution of the state $n$ to the specific heat. This is

$$
c_{V}=\frac{\mathrm{d}}{\mathrm{d} T} E_{n}(T)
$$


here the assumption of a constant volume $V$ implies a constant box length $L$. We obtain

$$
c_{V}=\mathrm{e}^{\frac{-n h v_{n}}{k T}} n h v_{n} \frac{\mathrm{d}}{\mathrm{d} T}\left(-\frac{n h v_{n}}{k T}\right)=\mathrm{e}^{\frac{-n h v_{n}}{k T}}\left(n h v_{n}\right)^{2} \frac{1}{k T^{2}}=k \mathrm{e}^{-u} u^{2}
$$

where

$$
u=\frac{n h v_{n}}{k T}
$$

$k$ is the Boltzmann constant.

\section{Examination of $c_{V}$}

The function (17) gives

$$
\frac{c_{V}}{k}=f(u)=\mathrm{e}^{-u} u^{2} .
$$

The first derivative of $f(u)$ is

$$
f^{\prime}(u)=\mathrm{e}^{-u}\left(2 u-u^{2}\right)
$$

which gives

$$
f^{\prime}(u)=0
$$

for

$$
u=u_{1}=0
$$

and

$$
u=u_{2}=2 \text {. }
$$

The second derivative of (19) is

$$
f^{\prime \prime}(u)=\mathrm{e}^{-u}\left(u^{2}-4 n+2\right)
$$

which for $u_{1}$ in (21a) becomes

$$
f^{\prime \prime}\left(u_{1}\right)=2>0
$$

indicating a minimum of $f(u)$ equal to

$$
f\left(u_{1}\right)=f(0)=0 .
$$

For $u_{2}$ in (21b) we have

$$
f^{\prime \prime}\left(u_{2}\right)=-2 \mathrm{e}^{-2}<0
$$

which indicates a maximum of $f(u)$ equal to

$$
f\left(u_{2}\right)=f(2)=4 \mathrm{e}^{-2} .
$$

A characteristic point is that results (19)-(24) hold for any quantum number $n$. The result

$$
u_{2}=2=\frac{n h v_{n}}{k T_{n}}
$$

implies that $T=T_{n}$ at the maxima of $c_{V}$ satisfy the equation 


$$
T_{n}=\frac{n h v_{n}}{2 k}=\frac{n^{2} h^{2}}{8 m L^{2} k}
$$

with the size of each maximum

$$
c_{V}^{\max }=k f\left(u_{2}\right)=k f(2)=k \frac{4}{e^{2}}
$$

independent of $n$. A similar property of the independence on $n$ holds for the minima of $c_{V}$ which are

$$
c_{V}^{\min }=k f\left(u_{1}\right)=k f(0)=0
$$

for all states $n$.

The formula (26) provides us with an important relation:

$$
k T_{n}=\frac{n^{2} h^{2}}{8 m L^{2}}=E_{n} .
$$

\section{Heat Transfer Due to the Temperature Interval $\Delta T$ between Two Extrema of the Specific Heat}

The heat transfer of the amount $\Delta E^{(h)}$ due to the temperature interval $\Delta T$ will be considered with the aid of a simplified formula

$$
\Delta E^{(h)}=c_{V} \Delta T
$$

where $c_{V}$ is, first, an average specific heat

$$
c_{V}=c_{V}^{a v}
$$

in the interval $\Delta T$ between two extrema (maxima) of the specific heat. In the second case $c_{V}$ is put equal to a maximum of the specific heat, i.e.

$$
c_{V}=c_{V}^{\max },
$$

where $c_{V}^{\max }$ is equal to the value obtained in formula (27). The both $\Delta E^{(h)}$, obtained respectively according to (29), (30) and (29), (31), will be next compared with the energy $\Delta E$ calculated from the energy difference between two quantum levels.

The temperature difference between two maxima of $c_{V}$ can be derived from the formula (26):

$$
\Delta T=T_{n+1}-T_{n}=\frac{(n+1)^{2}-n^{2}}{8 m L^{2} k} h^{2} .
$$

The average $c_{V}$ between these maxima is approximately equal to

$$
\frac{2 k}{2 \times 2} \int_{0}^{2} \mathrm{e}^{-u} u^{2} \mathrm{~d} u=\frac{1}{2} k \int_{0}^{2} \mathrm{e}^{-u} u^{2} \mathrm{~d} u
$$

where we assumed that the distance separating the maxima is roughly equal to a double distance between position of a minimum $(u=0)$ and that of the nearest maximum $(u=2)$ of $c_{V}$. In effect we obtain

$$
c_{V}^{a v}=\frac{1}{2} k \int_{0}^{2} \mathrm{e}^{-u} u^{2} \mathrm{~d} u=\left.\frac{k}{2}(-1) \mathrm{e}^{-u}\left(u^{2}+2 u+1\right)\right|_{u=0} ^{u=2}=\frac{1}{2} k 0.65 \approx k 0.33
$$


from which

$$
\Delta E^{(h)}=\frac{1}{2} k 0.65 \Delta T \approx 0.33 \frac{(n+1)^{2}-n^{2}}{8 m L^{2}} h^{2}=0.33 \Delta E_{n} .
$$

In the last step of (35) the energy difference between the electron states $n+1$ and $n$, viz.

$$
\Delta E_{n}=\frac{(n+1)^{2}-n^{2}}{8 m L^{2}} h^{2}
$$

obtained from (1), is taken into account.

The case of $c_{V}=c_{V}^{\max }$ leads to result

$$
\Delta E^{(h)}=k c_{V}^{\max } \Delta T=k \frac{2^{2}}{\mathrm{e}^{2}} \frac{(n+1)^{2}-n^{2}}{8 m L^{2} k} h^{2}=\left(\frac{2}{\mathrm{e}}\right)^{2} \Delta E_{n} \approx 0.54 \Delta E_{n} .
$$

which is not very much different from that obtained in (35).

\section{Change of Entropy Referred to the Energy Change of a Quantum System}

A well-known fact is that many ideas of classical physics did penetrate gradually into the quantum theory after being "only" submitted to modifications of a specific kind. The main idea of the modern theory was just to point out that physical parameters are quantum parameters. In general this means that a suitable size of parameter should be important for the physics of many phenomena in which the quantum situation can be involved.

The size limitations of the quantum theory did apply certainly to the energy and its changes. The aim of the present section and Secs. 8 and 10 is to examine from the quantum point of view the entropy parameter $S$ systematically accompanying the changes of energy in thermodynamics according to the formula [1]

$$
\mathrm{d} U+p \mathrm{~d} V=T \mathrm{~d} S
$$

here $U$ is the internal energy of a system having the volume $V$, the external pressure $p$ is exerted on $V$, and $T$ is temperature.

Because of the presence of $T$ and $p$ the system is regularly considered as a many-particle ensemble submitted to the laws of a statistical theory. However, a difficulty connected with a many-particle approach can be circumvented by limiting the problem to that similar to a one-particle system. This is easy to demonstrate on the example of a single particle enclosed in a potential box by considering the particle, say an electron, as a de Broglie wave of matter spread into a one-dimensional box volume of the length $L$.

In order to meet a quantum situation we should assume that the de Broglie wave, corresponding to any quantum state $n$ of the electron, has its special frequency $v_{n}$. When the number of free electrons in the box is reduced to a pair occupying the same quantum state $n$, it can be demonstrated that the kinetic energy of such pair, i.e. obtained by neglecting the electrostatic interaction energy, is equal to (see [8]): 


$$
n h v_{n}=2 E_{n}=\frac{n^{2} h^{2}}{4 m L^{2}} .
$$

A characteristic point is that a superposition of $n$ de Broglie waves of the same frequency $v_{n}$ gives a conservation of the kinetic energy of the pair when this pair is transformed into the waves; here

$$
h v_{n}
$$

is the energy carried by a single wave of the frequency

$$
v_{n}=\frac{n h}{4 m L^{2}} \text {. }
$$

The reciprocal value of $v_{n}$, viz.

$$
\tau_{n}=\frac{1}{v_{n}}=\frac{4 m L^{2}}{n h}
$$

is the time period of the electron wave equal to the oscillation time necessary for the electron to travel twice-in two opposite directions-along the box length $L$.

\section{Entropy Change and the Specific Heat}

The formula (38) can be simplified by assuming that the volume increment $\mathrm{d} V$ is equal to zero, i.e. we can put

$$
\Delta V=\Delta L=0 .
$$

In this case the entropy increment $\mathrm{d} S$ in (38) is reduced to

$$
\mathrm{d} U=T \mathrm{~d} S \text {. }
$$

Our aim becomes first to calculate a suitable specific heat $c_{V}$.

In Sec. 4 (see (14)) the problem-for the sake of simplicity-was reduced to a situation of a very low $T$ by limiting the Planck's probability expression for the temperature bath of $T$

$$
w_{n}=\mathrm{e}^{-\frac{n h v_{n}}{k T}}\left(1-\mathrm{e}^{-\frac{h v_{n}}{k T}}\right)
$$

to the first component entering (14). In this case the formula (17) accompanied by (18) is obtained.

In the present case we apply a full probability expression (44), therefore

$$
c_{V}^{\text {tot }}=c_{V}+c_{V}^{\text {suppl }}
$$

so $c_{V}$ calculated in (17) is supplemented by the expression

$$
c_{V}^{\text {suppl }}=-\mathrm{e}^{(n+1) h v_{n} / k T} n h v_{n}(n+1) h v_{n} \frac{1}{k T^{2}}=-\mathrm{e}^{-\frac{u}{n}(n+1)} \frac{u}{n}(n+1) u k .
$$

In result

$$
c_{V}^{\mathrm{tot}}=k \mathrm{e}^{-u} u^{2}\left[1-\mathrm{e}^{-u / n}\left(1+\frac{1}{n}\right)\right] .
$$

In the next step we examine the extremum position of $c_{V}^{\text {tot }}$ divided by $k$. To 
this purpose we calculate

$$
\begin{aligned}
\frac{\mathrm{d}}{\mathrm{d} u}\left(c_{V}^{\mathrm{tot}} / k\right) & =\frac{\mathrm{d}}{\mathrm{d} u} \mathrm{e}^{-u} u^{2}\left[1-\mathrm{e}^{-u / n}\left(1+\frac{1}{n}\right)\right] \\
& =\left(-\mathrm{e}^{-u} u^{2}+2 \mathrm{e}^{-u} u\right)\left[1-\mathrm{e}^{-u / n}\left(1+\frac{1}{n}\right)\right]+\mathrm{e}^{-u} u^{2}\left[-\frac{1}{n} \mathrm{e}^{-u / n}\left(1+\frac{1}{n}\right)\right] \\
& \cong \mathrm{e}^{-u}\left(2 u-u^{2}\right)\left(1-\mathrm{e}^{-u / n}\right)+\mathrm{e}^{-u} u^{2}\left(-\frac{1}{n} \mathrm{e}^{-u / n}\right) \\
& \cong \mathrm{e}^{-u}\left(2 u-u^{2}\right)\left(1-1+\frac{u}{n}\right)+\mathrm{e}^{-u} u^{2}\left(-\frac{1}{n}+\frac{u}{n^{2}}\right) \\
& \cong \mathrm{e}^{-u}\left(2 u-u^{2}\right) \frac{u}{n}-\mathrm{e}^{-u} u^{2} \frac{1}{n} \cong \mathrm{e}^{-u} u^{2} \frac{1}{n}(2-u-1)=0
\end{aligned}
$$

In the end steps of calculations we assumed that $n$ is large.

The extremal positions are obtained from (48) either for

$$
u=u_{1}=0
$$

or

$$
u=u_{2}=1
$$

The next derivative with respect to $u$ performed with the expression in (48) gives

$$
\frac{\mathrm{d}}{\mathrm{d} u} \mathrm{e}^{-u} u^{2}(1-u)=-\mathrm{e}^{-u} u^{2}(1-u)+\mathrm{e}^{-u}\left(2 u-3 u^{2}\right)
$$

Evidently for $u=u_{1}=0$ the formula (51) gives again zero, but a similar substitution of $u_{2}$ into (51) indicates a negative result:

$$
\left.\frac{\mathrm{d}}{\mathrm{d} u} \mathrm{e}^{-u} u^{2}(1-u)\right|_{u=1}=\mathrm{e}^{-1}(2-3)=-\frac{1}{\mathrm{e}}<0 ;
$$

in effect the property of a maximum position can be attributed to $u_{2}$.

By assuming the approximation

$$
1+1 / n \cong 1
$$

we obtain for $u=u_{2}=1$ the maximum value of the specific heat equal to

$$
c_{V}^{\mathrm{tot}}=\left.k \mathrm{e}^{-u} u^{2}\left(1-\mathrm{e}^{-\frac{u}{n}}\right)\right|_{u=1}=k \frac{1}{\mathrm{e}}\left(1-\frac{1}{\mathrm{e}^{1 / n}}\right)
$$

\section{De Broglie Waves for the Electron Particle in a Potential Box Referred to a Harmonic Oscillator}

Before the results for $c_{V}^{\text {tot }}$ are applied to calculations of the entropy let us demonstrate a harmonic-like behaviour of the de Broglie oscillating waves. This is based on an analysis of the oscillation constant together with the frequency and energy carried by the oscillator [9].

For a particle enclosed in a potential box of the length $L$ the amplitude $a$ of the de Broglie wave can be assumed to be close to $L$, i.e.

$$
a \approx L \text {, }
$$


so $a$ approaches the length of path allowed for the particle motion in one direction. This holds for any state $n$. The energy $W_{n}$ of the oscillator in state $n$ is coupled with $a$ and the oscillation constant $k_{n}$ by the formula [9]

$$
W_{n}=\frac{k_{n} a^{2}}{2} .
$$

In the next step $k_{n}$ is coupled with the frequency $v_{n}$ of the oscillator by the relation

$$
\omega_{n}=2 \pi v_{n}=\sqrt{\frac{k_{n}}{m}} .
$$

Together with (55) and (56) this implies that the formula

$$
a=L=\left(\frac{2 W_{n}}{k_{n}}\right)^{1 / 2}
$$

should be satisfied. Having

$$
n h v_{n}=W_{n},
$$

and the oscillator energy and particle energy formulae [see (7)] giving

$$
2 \pi v_{n}=2 \pi \frac{n h}{4 m L^{2}}=\frac{\pi}{2} \frac{n h}{m L^{2}},
$$

we obtain [see (56a), (57) and (59)]:

$$
\frac{k_{n}}{m}=\frac{2 W_{n}}{a^{2} m}=\frac{2 n h v_{n}}{L^{2} m}=\frac{2 n^{2} h^{2}}{4 m L^{2}} \frac{1}{L^{2} m}=\frac{n^{2} h^{2}}{2 m^{2} L^{4}} .
$$

On the other side from (57)

$$
\frac{k_{n}}{m}=4 \pi^{2} v_{n}^{2}
$$

which gives [see (59)]

$$
\frac{k_{n}}{m}=4 \pi^{2} \frac{n^{2} h^{2}}{16 m^{2} L^{4}}=\frac{\pi^{2}}{4} \frac{n^{2} h^{2}}{m^{2} L^{4}}
$$

valid for any $n$. Evidently the last formula in (62) differs only slightly from the result obtained in (60).

\section{Entropy Change Calculated with the Aid of the Specific Heat}

We calculate the entropy change by applying to it a maximal value of the specific heat. In this case [see (54)]:

$$
c_{V}^{\mathrm{tot}}=\left.k \mathrm{e}^{-u} u^{2}\left(1-\mathrm{e}^{-u / n}\right)\right|_{u=1} \cong k \frac{1}{\mathrm{e}}\left(1-1+\frac{1}{n}\right)=\frac{k}{n \mathrm{e}}
$$

since $u=u_{2}=1$ corresponds with a maximum value of $c_{V}^{\text {tot }}$.

Moreover in this case [see (18)]

$$
u=1=\frac{n h v_{n}}{k T_{n}},
$$

so we obtain [see (40)] 


$$
T_{n}=\frac{n h v_{n}}{k}=\frac{1}{k} \frac{n^{2} h^{2}}{4 m L^{2}}
$$

or

$$
n h v_{n}=k T_{n} .
$$

According to the formula (43) (see also [1]) we have

$$
\Delta S=\frac{\Delta U}{T}=c_{V}^{\text {tot }} \frac{\Delta T}{T} .
$$

Let us take the $\Delta T$ corresponding to the temperature difference between two neighbouring quantum states:

$$
\Delta T=T_{n+1}-T_{n}=\left[(n+1) h v_{n+1}-n h v_{n}\right] \frac{1}{k} .
$$

Therefore a maximal change of entropy corresponding to the change of the quantum number $n$ by one is

$$
\Delta S=c_{V}^{\text {tot }}\left[(n+1)^{2} \frac{h^{2}}{4 m L^{2}}-n^{2} \frac{h^{2}}{4 m L^{2}}\right] \frac{1}{k} \frac{4 m L^{2} k}{n^{2} h^{2}} \cong \frac{k}{n e} \frac{2 n}{n^{2}}=\frac{2 k}{e n^{2}}
$$

which implies

$$
\Delta S \sim \frac{1}{n^{2}}
$$

Let us note that $\Delta T$ between the neighbouring states obtained in virtue of (65) is twice as large as $\Delta T$ calculated in (32) with the aid of (26).

\section{Classical Physical Parameters Associated with Electron Transitions in Small Quantum Systems}

In many cases the treatment of quantum systems is considered as fundamentally separated from the classical physics and its results. Sometimes this view is dictated by the properties which are actually in our interest and are expected to belong mainly to a quantum domain. For example the statistics of the electron gas is usually a priori considered to be of a special character dictated by the Fermion-like classification of the electron particles and consequently, such kind of behaviour is from the beginning taken into account in course of the calculations performed on a given system. Another example concerns small quantum objects in which a special kind of the observable, say the energy connected with an electron transition, is mainly of interest. In this case the energy change due to transition is usually examined independently of other parameters which can be associated with the location of the electron particle and its change. In effect we obtain an exact or almost exact quantum result concerning the energy, but simultaneously an insight into numerous parameters describing the physical background of the transition process can be lost.

The aim of Sections 12 and 13 is to obtain some view on the classical secondary effects connected with the transition of an electron coupled mainly with the energy emission done in small quantum systems. 


\section{Transition Time between Quantum States and Its Reference to Parameters of the Classical Electrodynamics}

In quantum mechanics the transition time $\Delta t$ of an electron between two quantum energy levels is regularly difficult to assess. In fact the calculation of $\Delta t$ is replaced by a probabilistic treatment of the electron transitions started by Einstein [2] already in reference to the old quantum theory. A step due to the modern quantum theory was to associate the probabilities of the electron transitions with the matrix elements of the electron transition operator calculated with the aid of the wave functions describing both the initial and final state of the examined transition; see e.g. [10] [11]. An evident advantage was the selection rules for transitions provided by the calculation of the mentioned matrix elements, as well as the dependence of these rules on the character of the applied operator. A drawback seemed to be the absence of a direct insight into the size of the interval $\Delta t$.

This difficulty could be at least partly removed by applying the classical Joule-Lenz law for the transition of energy between the quantum levels [4] [12]. If we have the energy difference $\Delta E$ defined by the formula

$$
\Delta E=E_{n+1}-E_{n},
$$

so $\Delta E$ concerns a difference between two neighbouring quantum levels, the Joule-Lenz law states the relation [3] [4] [12]

$$
\frac{\Delta E}{\Delta t}=R i^{2} .
$$

here $R$ can be considered as a resistance due to the voltage $V$ connected with $\Delta E$ and intensity $i$ of the electron current between the states $n+1$ and $n$.

In other words we assume $i$ to be associated with a condenser having the voltage

$$
V=\frac{\Delta E}{e}
$$

and the current in the condenser is

$$
i=\frac{e}{t},
$$

where $t$ is the time of the current effectiveness. Together with the well-known formula

$$
R=\frac{V}{i}
$$

we have for (71) on the basis of (72)-(74) the result

$$
\frac{\Delta E}{\Delta t}=\frac{\Delta E}{e} \cdot \frac{t}{e}\left(\frac{e}{t}\right)^{2}=\frac{\Delta E}{t}
$$

which means that 


$$
\Delta t=t
$$

The $\Delta E$ in (72) and (75) is a parameter usually easy to obtain, whereas (76) can be associated classically with the resistance $R$ in (74) and the capacitance $C$ of the condenser by the formula

$$
t \approx R C
$$

see e.g. [13]. We expect (77) to be a constant term. In fact $C$ in (77) is given by the formula

$$
C=\frac{e}{V}=\frac{e^{2}}{\Delta E}
$$

whereas $R$ in virtue of (72) and (74) becomes

$$
R=\frac{\Delta E}{e} \cdot \frac{t}{e}=\frac{\Delta E t}{e^{2}} .
$$

In effect we obtain

$$
R C=t
$$

which is a confirmation of the expression given in (77).

This means that $R$ and $C$ should be known parameters in order to give the transition time (80). Beginning with $R$ we have

$$
R=\frac{V}{i}=\frac{\Delta E}{e} \cdot \frac{t}{e}=\frac{\Delta E \Delta t}{e^{2}}
$$

where in the last step the result of (76) is applied. We postulate that

$$
\Delta E \Delta t=h
$$

which implies that $t$ present in (77) should be a very special interval of time. This makes on the basis of (81)

$$
R=\frac{h}{e^{2}}
$$

which is a result much independent of the examined quantum system; see [4]. The validity of the postulate in (82) is checked in Sec. 13 given below.

A consequence of the formula (83) is that $R$ becomes equal to a well-known resistance value characteristic for the integer quantum Hall effect; see e.g. [14]. With the formula (83) for $R$ and (78) for $C$ we obtain

$$
R C=\frac{h}{\Delta E}
$$

or

$$
R C \Delta E=h \text {. }
$$

\section{The Time Interval Equal to $R C$ Characteristic for the Electron Transition in Different Quantum Systems}

We demonstrate an invariance of $R C$ in (84) for different quantum systems beginning with a free electron particle in a one-dimensional potential box. In this case (see e.g. [8]) 


$$
\Delta E=E_{n+1}-E_{n}=\frac{(n+1)^{2}-n^{2}}{8 m L^{2}} h^{2} \approx \frac{1}{4} \frac{n h^{2}}{m L^{2}}
$$

so from $(84)$

$$
R C=\frac{h}{\Delta E} \cong \frac{4 m L^{2}}{n h} .
$$

It should be noted that $R C$ in (87) is an interval equal to the time period characteristic for the free-electron oscillation in state $n$ :

$$
\tau_{n}=\frac{2 L}{v_{n}}=\frac{2 L}{n h} 2 m L=\frac{4 m L^{2}}{n h} .
$$

This holds because for a free electron in state $n$ the one-dimensional kinetic energy becomes

$$
E_{n}=\frac{m v_{n}^{2}}{2}=\frac{n^{2} h^{2}}{8 m L^{2}}
$$

from which we obtain the electron velocity in $n$ equal to:

$$
v_{n}=\left(\frac{n^{2} h^{2}}{4 m L^{2}}\right)^{1 / 2}=\frac{n h}{2 m L} ;
$$

see also (6). The result obtained in (90) is applied in (88).

A corresponding $R C$ can be calculated also for the electron transition in the hydrogen atom. By the virial theorem, we have the kinetic electron energy equal to the absolute value of the total electron energy:

$$
\frac{m v_{n}^{2}}{2}=\left|E_{n}\right|=\frac{m}{2}\left(\frac{e^{2}}{n \hbar}\right)^{2}=\frac{m e^{4}}{2 n^{2} \hbar^{2}} .
$$

here

$$
v_{n}=\frac{e^{2}}{n \hbar}
$$

is the electron velocity on the $n$th orbit; see [15]. The energy difference in the hydrogen atom is

$$
\begin{aligned}
\Delta E & =E_{n+1}-E_{n}=\frac{m}{2} \frac{e^{4}}{\hbar^{2}}\left[\frac{1}{n^{2}}-\frac{1}{(n+1)^{2}}\right] \\
& =\frac{m e^{4}}{2 \hbar^{2}} \frac{(n+1)^{2}-n^{2}}{n^{2}(n+1)^{2}} \cong \frac{m e^{4}}{2 \hbar^{2}} \frac{2 n}{n^{4}}=\frac{m e^{4}}{\hbar^{2}} \frac{1}{n^{3}},
\end{aligned}
$$

where in the last step $\Delta E$ is simplified to the case of large $n$. The time period of the electron circulation along the orbit $n$ becomes [15]

$$
\tau_{n}=\frac{2 \pi r_{n}}{v_{n}}=\frac{2 \pi n^{2} \hbar^{2}}{m e^{2}} \frac{n \hbar}{e^{2}}=\frac{2 \pi n^{3} \hbar^{3}}{m e^{4}},
$$

if we note that the radius $r_{n}$ of the $n$th circular orbit in the hydrogen atom is equal to [15] 


$$
r_{n}=\frac{n^{2} \hbar^{2}}{m e^{2}} .
$$

On the other hand from the formula (84) for RC and (93) for $\Delta E$ we obtain

$$
R C=\frac{h}{\Delta E}=\frac{h}{m e^{4}} \hbar^{2} n^{3}=\frac{2 \pi \hbar^{3} n^{3}}{m e^{4}} .
$$

This means that $R C$ becomes equal to the time period $\tau_{n}$ of the electron circulation given in (94).

The last small quantum system considered in the paper is the harmonic oscillator. For the oscillator having the frequency $v$ we have the time period of the oscillation equal to

$$
\frac{1}{v}=T
$$

and the energy difference between two neighbouring quantum levels is

$$
\Delta E=h v=\frac{h}{T}
$$

This gives

$$
R C=\frac{h}{\Delta E}=\frac{1}{v}=T .
$$

In effect the formula (84) is satisfied for all examined cases, i.e. the electron particle in a one-dimensional potential box, the electron in a hydrogen atom and the harmonic oscillator. In the last case the electron mass $m$ and charge $e$ do not enter into an explicit calculation of $R C$.

\section{Parameters of the Electron Mechanical Inertia Examined as a Check of the Formulae Applied for the Electron Transition}

Interesting results gives the examination of parameters connected with the electron inertia exhibited in course of the electron transitions, say those represented by the change

$$
n+1 \rightarrow n
$$

A similar reasoning was applied in considering the Tolman-Stewart effect characteristic for the slowing down of the motion of the electrons in metals; see e.g. [12] [13].

The energy difference

$$
\Delta E=e V
$$

can be represented by the product

$$
m a l=e V
$$

where $m$ is the electron mass, $a$-electron acceleration attained in course of the transition between the quantum levels, and $l$-the way length connected with the time characteristic for the transition. By the conservation of momentum this $l$ should be not much different than a distance travelled by an electron with the 
speed $v_{n}$ characteristic for the electron level at the end of transition multiplied by the transition time which is

$$
R C=\tau ;
$$

see respectively (88), (96) and (99) in different physical objects.

In effect we obtain

$$
l_{n}=2 L
$$

for a free particle in a one-dimensional potential box,

$$
l_{n}=2 \pi r_{n}
$$

in the hydrogen atom, and for the harmonic oscillator $l_{n}$ should approximate four times the amplitude of the classical oscillator equal to the product of the maximal velocity in state $n$ and time $\tau$ :

$$
l_{n}=v_{n}^{\max } \tau .
$$

In all these cases the acceleration $a$ in (102) can be represented by the ratio

$$
a=\frac{\Delta v}{\tau}
$$

where $\Delta v$ is the velocity change of the electron particle given by the difference

$$
\Delta v=v_{n+1}-v_{n} .
$$

In effect we obtain in place of (102) the equation

$$
m_{n} \Delta v=\tau e V=R C e V=\frac{V}{i} \frac{e}{V} e V=e^{2} \frac{V}{i}=e^{2} R=h,
$$

because of the result (83) obtained for $R$.

We check below that in fact equation (108) is well satisfied.

For a free-electron particle in the box:

$$
l_{n}=2 L
$$

is independent of $n$ and

$$
\Delta v=v_{n+1}-v_{n}=(n+1-n) \frac{h}{2 m L}=\frac{h}{2 m L} ;
$$

see (90). The above two formulae give

$$
m l_{n} \Delta v=m 2 L \frac{h}{2 m L}=h .
$$

For the hydrogen atomic orbit we have

$$
l_{n}=2 \pi r_{n}=2 \pi \frac{n^{2} \hbar^{2}}{m e^{2}}
$$

and the absolute value of $\Delta v$ is

$$
|\Delta v|=v_{n}-v_{n+1}=\frac{e^{2}}{n \hbar}-\frac{e^{2}}{(n+1) \hbar} \cong \frac{e^{2}}{n^{2} \hbar} .
$$

In this case the product entering the numerator of (102) becomes:

$$
m l_{n}|\Delta v|=m 2 \pi \frac{n^{2} \hbar^{2}}{m e^{2}} \frac{e^{2}}{n^{2} \hbar}=2 \pi \hbar=h .
$$


The above reasonings confirm the value (83) obtained for the resistance $R$.

The case of the harmonic oscillator is slightly more complicated [9]. The maximal electron velocity in state $n$ is given by the formula

$$
(1 / 2) m v_{\max }^{2}=n h v
$$

from which the distance $l$ obtained by the electron inertia in course of the time period of the electron oscillation $T$ is

$$
l=v_{\max } T=v_{\max } \frac{1}{v}=\frac{(2 n h)^{1 / 2}}{m^{1 / 2} v^{1 / 2}} .
$$

On the other hand the work performed by the electron on the distance $l$ is

$$
m l a=m l \frac{\Delta v_{\max }}{\tau}=e V
$$

where

$$
\tau=T
$$

is the time period connected with the electron current:

$$
i=\frac{e}{\tau}
$$

The $\Delta v^{\max }$ in (117) is a difference of the maximal velocities associated respectively with the quantum state $n+1$ and $n$ :

$\Delta v^{\max }=\left(\frac{2 h v}{m}\right)^{1 / 2}\left[(n+1)^{1 / 2}-n^{1 / 2}\right]=\left(\frac{2 h v}{m}\right)^{1 / 2} \frac{n+1-n}{(n+1)^{1 / 2}+n^{1 / 2}} \cong\left(\frac{2 h v}{m}\right)^{1 / 2} \frac{1}{2 n^{1 / 2}}$.

In effect we obtain for (117):

$$
m l \Delta v^{\max }=m \frac{(2 n h)^{1 / 2}}{m^{1 / 2} v^{1 / 2}}\left(\frac{2 h v}{m}\right)^{1 / 2} \frac{1}{2 n^{1 / 2}}=h
$$

which is the expected result.

\section{Summary}

In the first step, by assuming the conservation of energy, the electron states enclosed in a one-dimensional potential box are transformed into the de Broglie waves having definite frequencies in time. The dependence of these waves on temperature can be studied according to the well-known Planck's formalism applied to the black-body radiation. When the specific heats due to the electron waves are examined, they show an extremal (maximal) behaviour at some temperature different from the absolute zero. In a further treatment, the difference of temperature characteristic for two extremal positions of the specific heat obtained for two quantum levels can be multiplied by a maximal specific heat due to these levels. This product estimates the amount of the heat transfer of energy associated with the electron transition. The amount occurs to be close to the change of the electron energy due to the quantum-mechanical transition.

The next step of the paper concerns the calculation of the changes of the entropy of a free-electron particle enclosed in a potential box. Usually the temper- 
ature and entropy refer to a large ensemble of particles whose statistics and energy are known. However, at the early stage of the quantum theory, the particle ensembles were considered as sets of the oscillators having definite frequencies and remaining in equilibrium with respect to some external temperature. According to the de Broglie idea, it is easy to transform the behaviour of particles enclosed in a potential box into that of a system of the oscillating waves. In effect, the thermodynamical properties of the oscillators, like the specific heat, can be examined with the aid of the probabilistic formalism given by Planck. Because of the relation existent between the specific heat and entropy, the changes of entropy which accompany the energy transfer become easy to calculate with the aid of the temperature intervals defining the separations between the quantum levels.

In the last step, the aim of the paper was to point out that the time of the emission of energy between two neighbouring quantum states is associated-on a classical level-with production of the electric current due to a transport of a single electron particle. The current corresponds to a temporary formation of the electric cell in a quantum system and exhibits the properties of intensity and resistance known from the classical electrodynamics.

Having a known energy emission $\Delta E$, the current parameters provide us-on the basis of the Joule-Lenz law-with the time $\Delta t$ necessary for the dissipation of energy $\Delta E$. But the same interval $\Delta t$ can be obtained as a product of the current resistance $R$ and capacitance $C$ of the condenser represented by a temporary presence of the potential difference $\frac{\Delta E}{e}$ between two quantum levels.

The calculations are limited to three quantum objects considered as examples: the electron in a one-dimensional potential box, electron in a hydrogen atom and the electron representing a harmonic oscillator.

Classical parameters obtained for the examined objects in effect of the emission process are checked by considering the properties of the electron mechanical inertia exhibited in the same objects in course of the emission of the energy $\Delta E$.

\section{Conflicts of Interest}

The author declares no conflicts of interest regarding the publication of this paper.

\section{References}

[1] Planck, M. (1932) Einführung in die Theorie der Wärme. S. Hirzel, Leipzig.

[2] Einstein, A. (1917) Physikalische Zeitschrift, 18, 121.

[3] Lass, H. (1950) Vector and Tensor Analysis. McGraw-Hill, New York.

[4] Olszewski, S. (2015) Journal of Modern Physics, 6, 1277. ibid. (2016) 7, 162; (2017) Acta Physica Polonica, 131, 226.

[5] Sommerfeld, A. and Bethe, H. (1933) The Electron Theory of Metals. In H. Geiger 
\& K. Scheel (Eds.), Handbuch der Physik (Vol. 24, Part 2), Berlin: Springer. (In German)

[6] Mott, N.F. and Jones, H. (1936) The Theory of the Properties of Metals and Alloys. Oxford University Press, Oxford, New York.

[7] Ziman, J.M. (1972) Principles of the Theory of Solids. Cambridge University Press, UK. https://doi.org/10.1017/CBO9781139644075

[8] Eyring, H., Walter, J. and Kimball, G.E. (1957) Quantum Chemistry. Wiley, New York.

[9] Sommerfeld, A. (1949) Mechanik. 4th Ed., Akademische Verlagsgesellschaft, Leipzig.

[10] Schiff, L.I. (1968) Quantum Mechanics. 3rd Ed., McGraw-Hill, New York.

[11] Slater, J.C. (1960) Quantum Theory of the Atomic Structure. Vol. 2, McGraw-Hill, New York.

[12] Matveev, A.N. (1964) Electrodynamics and the Theory of Relativity. Izd, Wyzszaja Szkola, Moscow. (In Russian)

[13] Jauncey, G.E.M. (1948) Modern Physics. 3rd Ed., Van Nostrand, New York.

[14] MacDonald, A.H. (1989) Quantum Hall Effect. A Perspective, Kluwer, Milano. https://doi.org/10.1007/978-94-010-9709-3

[15] Sommerfeld, A. (1931) Atombau und Spektrallinien. 5th Ed., Vieweg, Braunschweig. 